\title{
Quantifying dynamic fire behaviour phenomena using Unmanned Aerial Vehicle technology
}

\author{
$\underline{\text { Alexander Filkov }}^{\text {a,b (iD), Brett Cirulis a and Trent Penman }}{ }^{a}$ \\ ${ }^{a}$ School of Ecosystem and Forest Sciences, University of Melbourne, Creswick, Victoria Australia, ${ }^{b}$ Bushfire \\ and Natural Hazards Cooperative Research Centre, Melbourne, Victoria Australia \\ Email: alexander.filkov@unimelb.edu.au
}

\begin{abstract}
Catastrophic wildfires are often a result of dynamic fire behaviours. Although some of these behaviours have been described and investigated, others require further study. Fire coalescence and junction fires are particular cases of merging fire fronts and are common phenomena observed during bushfires. They are important as they can cause rapid escalation of fire behaviour and be dangerous for ground-based emergency personnel. There are a few studies devoted to investigation of merging fires in field conditions. There is a need for high temporal and spatial measurements of fire behaviour in field conditions in order to better understand this phenomenon and evaluate risks during bushfires and prescribed burning. The aim of this study was to test emerging technologies for better quantification of fire behaviour at field scales and evaluate their potential as an operational tool.
\end{abstract}

Several small and medium scale field experiments were conducted during April 2019 on farmland in Victoria, Australia. Harvested wheat fields were used as experimental plots, as they form relatively homogeneous fuel beds. Fuel height varied from 18 to $40 \mathrm{~cm}$ with fuel load and moisture content at $1.1 \mathrm{t} / \mathrm{ha}$ and $11.9 \%$ respectively. Wind speed varied in the range of 1.5-6.5 m/s. An Unmanned Aerial Vehicle (UAV) was used to capture high definition video imagery of fire propagation in synchronisation with sensor data from the onboard Global Positioning System (GPS) and Inertial Measurement Unit (IMU). These sensors enabled the platform/camera orientation and position in space to be aligned with the video footage of fire propagation and to be georeferenced in GIS software.

Twenty-one junction fires and five inward parallel fire fronts (parts of the fire lines approaching each other) were identified during the experiments. The rate of spread (ROS) of merging fire fronts was found to be at least two times higher than for the basic fire fronts (the rate of spread of a linear fire front in the same fuel bed in no-slope conditions) and for junction fires with acute angles $\left(<14^{\circ}\right)$ it increased 6 times and more. Inward parallel fire fronts spread much slower, varying between 0.05 and $0.25 \mathrm{~m} / \mathrm{s}$. Forty-six percent of junction fires had increase of the ROS at the final stage of the merging process in contrast to Thomas et al. (2017) and Viegas et al. (2012). Also, it was observed that the angle between two oblique fire fronts did not change significantly in time for the initial angles smaller than $34^{\circ}$. It can be assumed that the main fire front influences on the shape and ROS respectively of junction fires and laboratory experiments cannot fully replicate these conditions.

Although the initial experimental conditions were very different in relation to scale, fuel and wind conditions, similar ROS to that shown in numerical simulations by Thomas et al. (2017) were observed in our field experiments. Further investigation is required to explain the similarities as the relationship between fuel load, wind speed and scale is not known. The comparison of corrected values of dimensionless ROS for different angles between fire fronts with laboratory experiments of Viegas et al. (2012) showed reasonable quantitative agreement.

These experiments have shown that the method of using UAV's to capture georeferenced video footage can be used reliably to quantify fire behaviour phenomena for research, operation and management purposes.

Keywords: Remote measurements, UAS, fire front propagation, merging fire fronts 
Filkov et al., Using technological advancements to uncover fire behaviour phenomena and for operational support

\section{INTRODUCTION}

Extreme fire events (Tedim et al. 2018) have become more regular around the world. Fighting them requires extensive human resources, and they can result in many casualties and loss of property. In most cases, these consequences are a result of dynamic fire behaviours (Filkov et al. 2018a, 2018b; Tedim et al. 2018; Viegas 2012). Although some have been described and investigated, there are others that require further study, such as fire coalescence and junction fires (or jump fires previously) (Hilton et al. 2018; Raposo et al. 2018; Thomas et al. 2017; Viegas et al. 2013; Viegas et al. 2012). The convergence of separate individual fires into larger fires is known as coalescence and can lead to rapid increases in fire intensity and spread rate (Hilton et al. 2017). Junction fires are the result of the merging of two lines of fire intersecting at an oblique angle (Viegas et al. 2012). Fire coalescence and junction fires are particular cases of the merging of fire fronts (Figure 1).

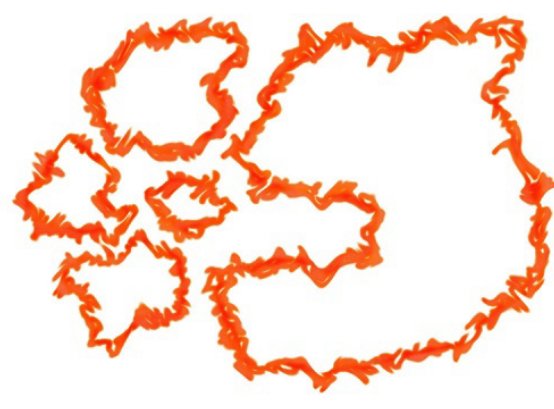

a)

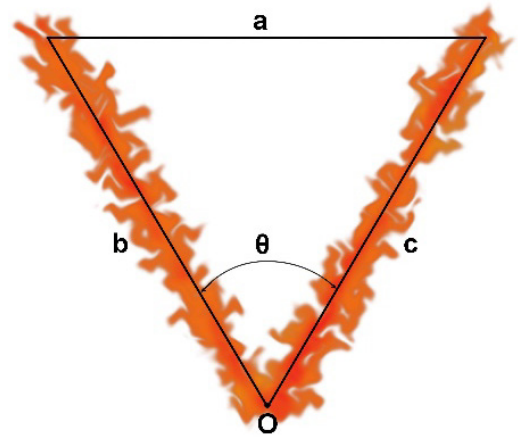

b)

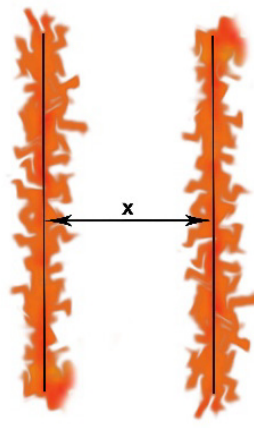

c

Figure 1. Merging fire fronts: a) Fire coalescence, b) Junction fire, c) Parallel fire fronts. Where $a, b$ and $c$ are the dimensions of the junction fire; $\theta$ is the angle between oblique fire fronts; $x$ is the distance between parallel fire fronts.

There is no clear answer what are the mechanisms that drive junction fires despite intensive investigation (Hilton et al. 2018; Raposo et al. 2018; Thomas et al. 2017; Viegas et al. 2013; Viegas et al. 2012). Viegas et al. (2012) started to study this phenomenon in the laboratory conducting numerous experiments. They varied the angle between two fire fronts (Viegas et al. 2012), slope and fuel type (Viegas et al. 2013). Recently they compared laboratory experiments with 3 field experiments and one real fire (Raposo et al. 2018). They determined two stages of junction fire development - acceleration and deceleration stages. They assumed that only in the final stage of fire deceleration radiation from the flames be considered the main mechanism of fire spread using the concept of propagation flux proposed by Rothermel.

Thomas et al. (2017) used the WRF-Fire coupled atmosphere-fire model to simulate the dynamic propagation of junction fires. They tested similar angles $\theta$ to Viegas et al. (2012), but at much bigger scale, each fire line was $1000 \mathrm{~m}$ long. They found that in addition to the bulk fire-induced surface flow, it includes the formation of counter-rotating pairs of vertical vortices lying on or ahead of the fireline, which result in acceleration of fire front propagation. They concluded that the vortical structures are not well resolved at 20-m resolution and are too small to be properly resolved by their simulations. Therefore, they did not get quantitative agreement with the Viegas et al. (2012) study.

(Hilton et al. 2018) developed a two-dimensional propagation model coupled to a 'pyrogenic' potential flow formulation to simulate merging fires. One of the assumptions of the model is that the plume is not significantly affected by the wind. This assumption is problematic as the wind tilts the flame, resulting in increased radiative heat flux on the fuel under the flame at higher wind speeds. The authors concluded that despite the good match to experimental results, the model should take flame attachment into account and it is likely that the model only applies under certain conditions which have not been fully explored in the experimental parameter space.

Recent study of Raposo et al. (2018) showed that laboratory experiments for low angles of junction fires cannot capture all effects due to the small scale. Therefore, high temporal and spatial measurements of wind field and radiative heat flux in the field conditions are required to capture effects of convection and radiation at different stages of merging fires development to improve our understanding of this phenomenon and inputs to operational fire behaviour models.

Unmanned Aerial Vehicles (UAV or drones) can be a useful tool in obtaining these measurements. In the last decade they have become well utilised in different areas, including the study of wildfires. UAVs have been used for fire detection and monitoring (Hua \& Shao 2017), fire management (Merino et al. 2012), and post fire 
Filkov et al., Using technological advancements to uncover fire behaviour phenomena and for operational support

monitoring (Fernández-Guisuraga et al. 2018). They can be equipped with various sensing instruments, ranging from optical sensors (including visible and infrared) to microwave sensors (Radar and Lidar). Owing to their flexibility, low cost, and high resolution data collection, rotary-wing drone remote sensing can fill data gaps about different fire behaviour phenomena.

The aim of this study was to test UAVs in combination with new software for fire behaviour characterization, specifically junction fires, and its applicability for operational support.

\section{METHODS}

\subsection{Study area and equipment}

The study was conducted on farmlands in western Victoria, Australia (Figure 2). Several preliminary small and medium scale field experiments were conducted on 12 $12^{\text {th }}$ of April 2019 near Kingston $110 \mathrm{~km}$ west of Melbourne.

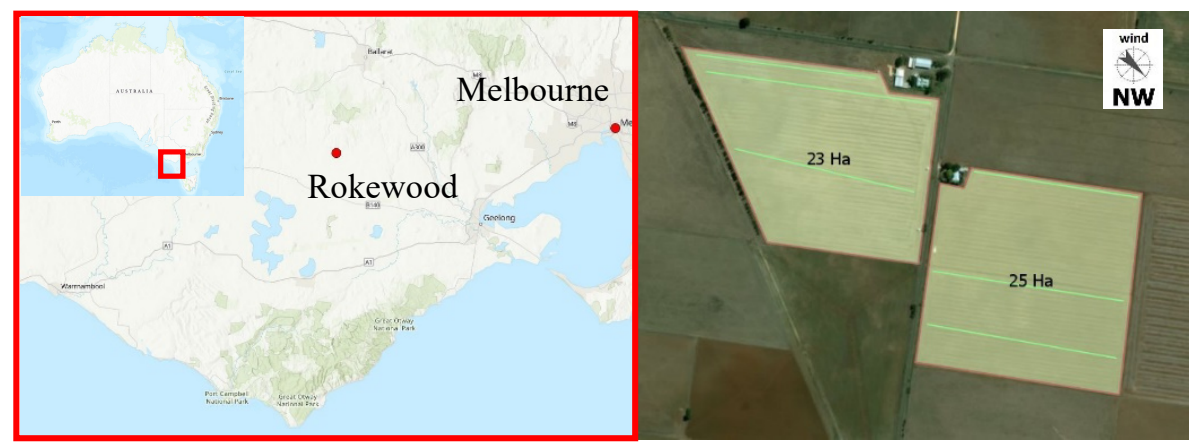

Figure 2. Location of experimental plots. Green lines represent ignition lines.

Harvested wheat fields were used as experimental plots, as they form relatively homogeneous fuel beds. Fuel height varied from 18 to $40 \mathrm{~cm}$ and fuel load and moisture content were $1.1 \mathrm{t} /$ ha and $11.9 \%$ respectively. Wind direction was mostly north westerly in the range of $1.5-6.5 \mathrm{~m} / \mathrm{s}$. The slope was minimal $\left(<5^{\circ}\right)$ at all plots. A DJI Mavic Pro (UAV) was used to capture high definition video imagery of fire propagation in synchronisation with sensor data from the on-board Global Positioning System (GPS) and Inertial Measurement Unit (IMU). These sensors enabled the platform/camera orientation and position in space to be aligned with the video footage and the fire propagation georeferenced in GIS software.

\subsection{Data capture and processing}

Video data was captured using the onboard camera on the DJI Mavic Pro. To minimise the georeferencing error of the final imagery, a flight altitude of $30 \mathrm{~m}$ and a $90^{\circ}$ camera angle were maintained whilst video footage was recorded. Video was recorded at a 1080p resolution at $60 \mathrm{fps}$. The CIRRUAS application was used with an android phone to record the necessary flight metadata for post processing (CompassDrone 2019). The post processing phase was completed for each video and metadata file using the Full Motion Video (FMV) toolbox within the ArcGIS Pro software (Macdonald 2017). Prior to analysing the video footage, the video file was converted into a FMV-compliant format. The metadata log file containing sensor information is combined with the video file in a process called Multiplexing. The result is a video file with each frame georeferenced. The multiplexed video file was then used to identify and spatially define fire fronts at set time intervals.

Line ignition of fuel was done downwind by a drip torch (50\% diesel fuel to $50 \%$ petrol) along the edge of the experimental plots (Figure 2). Six straight ignition lines were ignited during the experiment. After starting the ignition line, the fire front produced fire tongues. When the fire lines of two neighbouring tongues merged together, we identified it as a junction fire. Inward parallel fire fronts (parallel fire fronts hereafter) were identified when two fire tongues burnt parallel to each other.

To calculate ROS of merging fire fronts we measured travelling distance of intersect point $\mathrm{O}$ (Figure 1b) every 2 seconds. Only inward parallel fire fronts (parts of the fire lines approaching each other) were observed during the experiments. For parallel fire fronts, we randomly selected two points in the fire fronts in front of each other and measured the distance between them every 5 seconds. Basic ROS was measured in vicinity to each junction fire and parallel fire fronts every 5 seconds for their entire duration. Different time intervals of selected phenomena were chosen due to the significant distinction of time scales. Wind conditions were identical for each pair of linear fire front and junction fires, linear fire front and parallel fire fronts. 
Filkov et al., Using technological advancements to uncover fire behaviour phenomena and for operational support

To compare ROS between different phenomena and with other studies we used approach proposed by Viegas et al. (2012). They used non-dimensional form of the rate of spread of intersection point of two oblique fire fronts (merging fire fronts):

$$
V^{\prime}=\frac{V_{O}}{R_{0}}
$$

where $\mathrm{V}_{\mathrm{O}}$ is the rate of spread of intersection point of two oblique fire fronts; $\mathrm{R}_{0}$ is the basic rate of spread of a linear fire front, i.e. the rate of spread of a linear fire front in the same fuel bed in no-wind and no-slope conditions. The angle between linear fire front and wind direction varied in the range 45-135 degrees. In our particular case we measured $\mathrm{R}_{0}$ of a closest linear fire front in presence of wind to analysed merging fire fronts. This modification allows to exclude wind from the analysis and to conduct comparison in the future with other experiments at different weather conditions.

\section{RESULTS AND DISCUSSION}

Eleven video footages were filmed during experiments and post processed (Figure 3a). Twenty-six merging fires were identified, namely 21 junction fires and 5 parallel fire fronts.

It was found that all considered junction fires can be separated into 5 groups depending on the angle between oblique fire fronts $\theta: 4^{\circ}-14^{\circ}, 28^{\circ}-34^{\circ}, 40^{\circ}-59^{\circ}$ and above $76^{\circ}$. Highest number of fires $(43 \%)$ were observed in $28^{\circ}-34^{\circ}$ group.

Figure $3 \mathrm{~b}$ shows comparison of ROS of observed fire fronts.
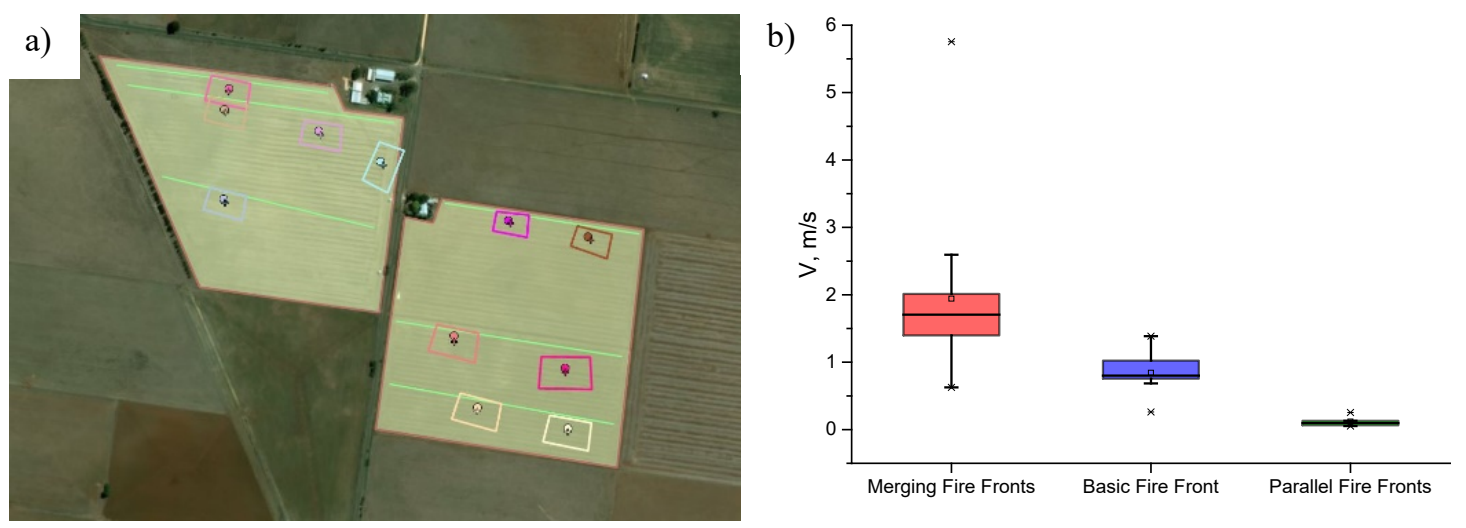

Figure 3. a) Bird view of video footages. Each rectangular represents separate video footage. b) Comparison of different types of fire front propagation.

ROS of merging, basic and parallel fire fronts was calculated as an average of all 2 and 5 second time intervals. It was observed that propagation of merging fire fronts was faster compared to other fire fronts, which is consistent with other studies (Viegas et al. 2013; Viegas et al. 2012). Median ROS of merging fire fronts was at least two times higher than for basic and parallel, $1.7 \mathrm{~m} / \mathrm{s}$ compare to 0.8 and $0.096 \mathrm{~m} / \mathrm{s}$ respectively. For acute angles $\left(<14^{\circ}\right)$ ROS of merging fires was greater than 6 times that of basic ROS. Parallel fire fronts spread much slower, varying between 0.05 and $0.25 \mathrm{~m} / \mathrm{s}$.

Comparison of $\theta$ and ROS for observed junction fires are presented on Figure 4.

As expected, it was observed that the more acute the angle the higher the ROS. Forty-six percent of junction fires had increase of the ROS at the final stage of the merging process in contrast to Thomas et al. (2017) and Viegas et al. (2012) (Figure 4b). It was also observed that for two oblique fire fronts with initial angles smaller than $34^{\circ}$, the angle between fire fronts did not increase significantly over time (Figure $4 \mathrm{a}$ ). It can be assumed that the main fire front influences on the shape of junction fires and ROS respectively and laboratory experiments cannot fully replicate these conditions. This requires further investigation. 
Filkov et al., Using technological advancements to uncover fire behaviour phenomena and for operational support
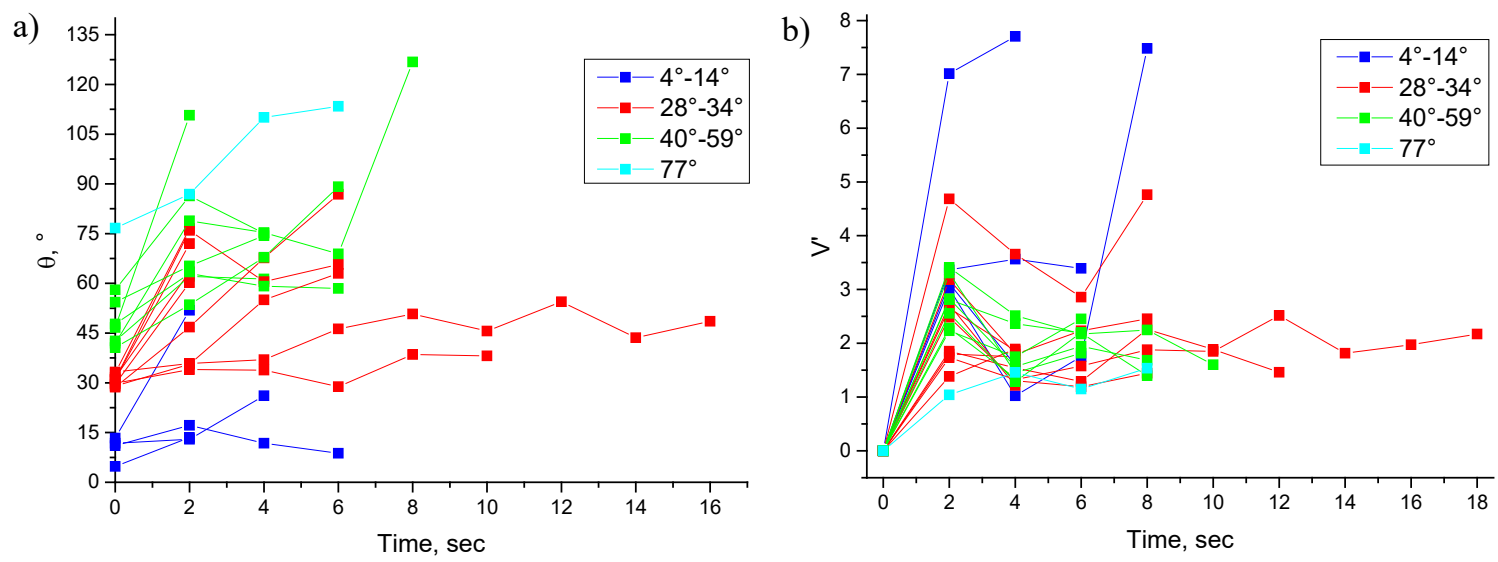

Figure 4. Evolution of fire fronts in time: a) variation of the angle $\theta$ between oblique fire fronts; b) variation of the dimensionless rate of spread $V^{\prime}$. Angles in the legend indicate the value at the initial time.

Two papers (Thomas et al. 2017; Viegas et al. 2012) presenting results on merging wildfires and relevant to our study were found in the literature. Both studies were conducted without wind. Neither Viegas et al. (2012) nor Thomas et al. (2017) mentioned moisture content of fuel bed in their studies.

Figures 5 and 6 show comparison of ROS with experimental and modelling results.

a)

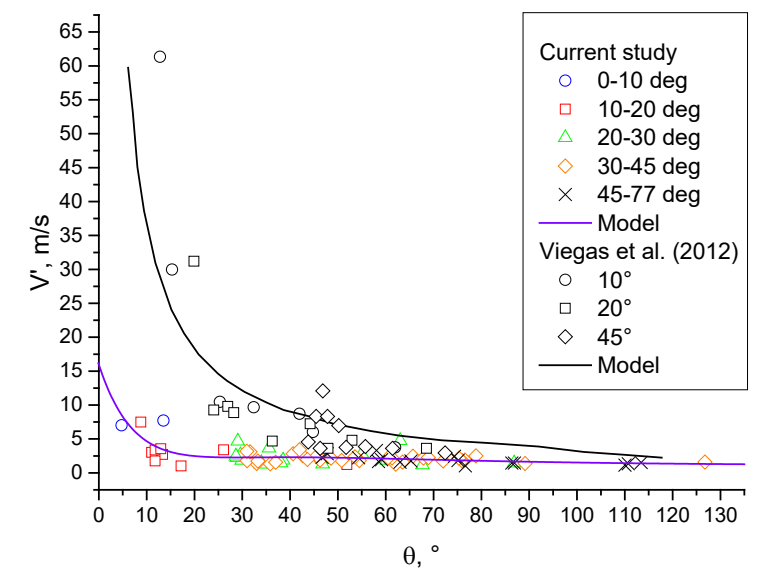

b)

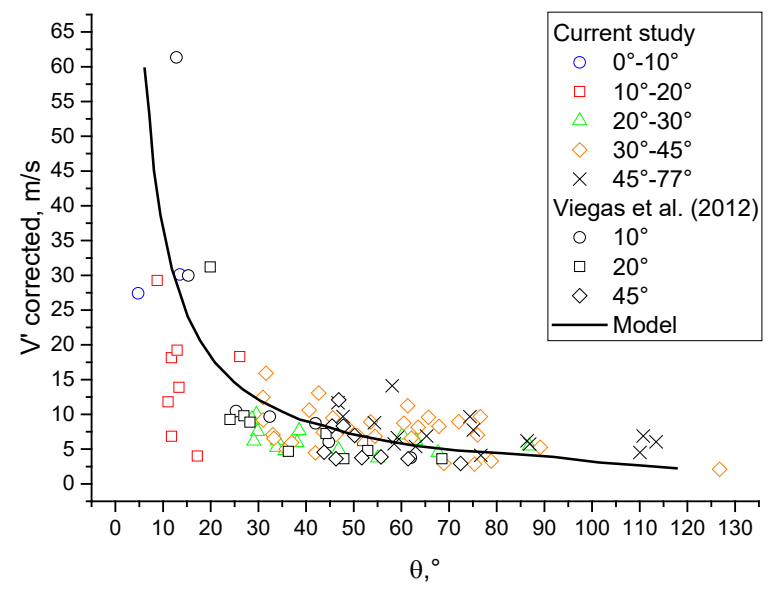

Figure 5. Comparison of dimensionless rate of spread $V^{\prime}$ with Viegas et al. (2012): a) original $V^{\prime}$; b) corrected V'. Markers indicate angle between merging fire fronts at different moments of time. Legend shows the ranges of initial angles $\theta$. Black line is the simplified analytical model of Viegas et al. (2012);

violet line is a regression line from current study, adjusted $R^{2}=0.99$. 
Filkov et al., Using technological advancements to uncover fire behaviour phenomena and for operational support

It can be seen from the Figure 5a that results differ. However, both datasets can be modelled with the exponential function. Nonlinear regression with Orthogonal Distance Regression algorithm was used to model our experimental data. The data were fitted using the following equation:

$$
V^{\prime}=1.08+3.85 * \exp (-(\theta-31.58) / 9.81)-5.04 * \exp (-(\theta-31.58) / 11.11)+2.4 * \exp (-(\theta-31.58) / 41.2)
$$

The difference can be related to different fuel load and structure, 6 times higher in Viegas et al. (2012). Also, basic ROS was calculated in presence of wind, unlike Viegas et al. (2012). To exclude this as a factor we recalculated $V^{\prime}$ using an average basic ROS of Viegas, which was $0.2 \mathrm{~m} / \mathrm{s}(\approx 1 \mathrm{~m} / \mathrm{s}$ in our study). Comparison of corrected values of $V$ ' with Viegas' experimental results is presented on Figure $5 \mathrm{~b}$. Analysis shows a good quantitative agreement with Viegas et al. (2012).

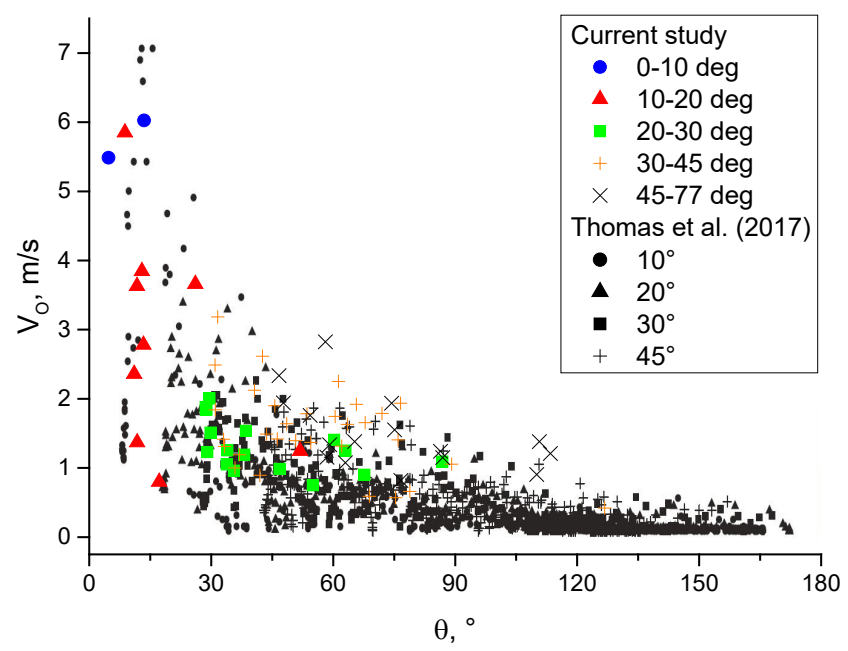

Figure 6. Comparison of the rate of spread of intersection point of two oblique fire fronts with Thomas et al. (2017). Markers indicate angle between merging fire fronts at different moments of time. Legend shows the ranges of initial angles $\theta$.

Thomas et al. (2017) conducted a comparison with experimental results of Viegas et al. (2012) as well and they could not get quantitative agreement with them. They assumed that the reason is the different scale of experiments and numerical modelling.

Analysis of the data on Figure 6 shows a good agreement with dataset of Thomas et al. (2017). However, it should be mentioned that fuel load in Thomas et al. (2017) was 7 times higher and the experiments conducted in no wind conditions. Without having the same initial conditions in both studies it is not possible to make any conclusion regarding influence of scale effect on the ROS of merging fire fronts. To address this issue the future research will focus on studying the scale effect in field conditions.

\section{CONCLUSION}

In this study, several preliminary small and medium scale field experiments were conducted in April 2019 on harvested wheat fields in Australia to test emerging technologies for fire behaviour characterisation and operational application. A UAV was used to capture high definition video imagery of fire propagation. Twentyone junction fires and five inward parallel fire fronts were identified during the experiments.

ROS of merging fire fronts $V_{O}$ was found to be at least two times higher than for the basic fire fronts and for acute angles $\left(<14^{\circ}\right)$ it increased by 6 times or more. Inward parallel fire fronts spread much slower, varying between 0.05 and $0.25 \mathrm{~m} / \mathrm{s}$. Forty-six percent of junction fires had increased in the ROS at the final stage of the merging process, a result in contrast to Thomas et al. (2017) and Viegas et al. (2012). It was also observed that the angle between two oblique fire fronts did not change significantly over time if the initial angle was smaller than $34^{\circ}$. It can be assumed that the main fire front influences on the shape and ROS respectively of junction fires and laboratory experiments cannot fully replicate these conditions. A good agreement was obtained with numerical modelling of Thomas et al. (2017), although we had very different initial conditions, such as smaller scale, fuel load and the presence of wind. Additional research is required to understand these results. Comparison of corrected values of dimensionless ROS $V$ ' for different angles between fire fronts with 
Filkov et al., Using technological advancements to uncover fire behaviour phenomena and for operational support

laboratory experiments of Viegas et al. (2012) showed good quantitative agreement, while fuel load in our tests was 7 times lower.

The quantification of captured video and photo imagery has traditionally been challenging and requires significant pre-experiment set up time or a complex post processing workflow. The approach used in these experiments has the benefit of minimal set up time with the resulting data being highly accurate across space and time. With further development and testing it shows promise to be a valuable tool for fire behavior research, operation and management applications.

\section{ACKNOWLEDGMENTS}

This study was funded by the Bushfire and Natural Hazards Cooperative Research Centre 'Determining threshold conditions for extreme fire behaviour' project. We thank John McKinnon for providing access to his farmland for the experiments.

\section{REFERENCES}

Compassdrone. (2019). CIRRUAS App (Version 1.0.4). Retrieved from https://compassdrone.com/software/cirruas-app/

Fernández-Guisuraga, J.M., Sanz-Ablanedo, E., Suárez-Seoane, S., Calvo, L. (2018). Using unmanned aerial vehicles in postfire vegetation survey campaigns through large and heterogeneous areas: Opportunities and challenges. Sensors, 18(2), 1-17.

Filkov, A.I., Duff, T.J., Penman, T.D. (2018a). Extreme fire behaviours: Surveying fire management staff to determine behaviour frequencies and importance. Paper presented at the Bushfire and Natural Hazards CRC \& AFAC conference, Perth, Australia, 5 - 8 September 2018, 1-5.

Filkov, A.I., Duff, T.J., Penman, T.D. (2018b). Improving fire behaviour data obtained from wildfires. Forests, 9, 1-21.

Hilton, J., Sharples, J., Sullivan, A., Swedosh, W. (2017). Simulation of spot fire coalescence with dynamic feedback. 22nd International Congress on Modelling and Simulation, Hobart, Tasmania, Australia, 3 to 8 December 2017.

Hilton, J.E., Sullivan, A.L., Swedosh, W., Sharples, J., Thomas, C. (2018). Incorporating convective feedback in wildfire simulations using pyrogenic potential. Environmental Modelling and Software, 107, 1224.

Hua, L., Shao, G. (2017). The progress of operational forest fire monitoring with infrared remote sensing. Journal of Forestry Research, 28(2), 215-229.

Macdonald, O. (2017). Getting to know ArcGIS Pro. Cartographic Journal, 54(3), 284-285.

Merino, L., Caballero, F., Martínez-De-Dios, J.R., Maza, I., Ollero, A. (2012). An unmanned aircraft system for automatic forest fire monitoring and measurement. Journal of Intelligent and Robotic Systems: Theory and Applications, 65(1-4), 533-548.

Raposo, J.R., Viegas, D.X., Xie, X., Almeida, M., Figueiredo, A.R., Porto, L., Sharples, J. (2018). Analysis of the physical processes associated with junction fires at laboratory and field scales. International Journal of Wildland Fire, 27(1), 52-68.

Tedim, F., Leone, V., Amraoui, M., Bouillon, C., Coughlan, R.M., Delogu, M.G., Fernandes, M.P., Ferreira, C., Mccaffrey, S., Mcgee, K.T., Parente, J., Paton, D., Pereira, G.M., Ribeiro, M.L., Viegas, D.X., Xanthopoulos, G. (2018). Defining Extreme Wildfire Events: Difficulties, Challenges, and Impacts. Fire, 1(9), 1-28.

Thomas, C.M., Sharples, J.J., Evans, J.P. (2017). Modelling the dynamic behaviour of junction fires with a coupled atmosphere-fire model. International Journal of Wildland Fire, 26(4), 331-344.

Extreme Fire Behaviour 1-56 (Nova Science Publishers, Inc. 2012).

Viegas, D.X., Raposo, J., Figueiredo, A. (2013). Preliminary analysis of slope and fuel bed effect on jump behavior in forest fires. Procedia Engineering, 62, 1032-1039.

Viegas, D.X., Raposo, J.R., Davim, D.A., Rossa, C.G. (2012). Study of the jump fire produced by the interaction of two oblique fire fronts. Part 1. Analytical model and validation with no-slope laboratory experiments. International Journal of Wildland Fire, 21(7), 843-856. 